BULLETIN (New Series) OF THE AMERICAN MATHEMATICAL SOCIETY

Volume 34, Number 4, October 1997, Pages 423-433

S 0273-0979(97)00731-3

\title{
NONLOCAL INVARIANTS IN INDEX THEORY
}

\author{
STEVEN ROSENBERG
}

\begin{abstract}
In its original form, the Atiyah-Singer Index Theorem equates two global quantities of a closed manifold, one analytic (the index of an elliptic operator) and one topological (a characteristic number). Because it relates invariants from different branches of mathematics, the Index Theorem has many applications and extensions to differential geometry, K-theory, mathematical physics, and other fields. This report focuses on advances in geometric aspects of index theory.

For operators naturally associated to a Riemannian metric on a closed manifold, the topological side of the Index Theorem can often be expressed as the integral of local (i.e. pointwise) curvature expression. We will first discuss these local refinements in $\S 1$, which arise naturally in heat equation proofs of the Index Theorem. In $\S \S 2,3$, we discuss further developments in index theory which lead to spectral invariants, the eta invariant and the determinant of an elliptic operator, that are definitely nonlocal. Finally, in $\S 4$ we point out some recent connections among these nonlocal invariants and classical index theory.
\end{abstract}

\section{LOCAL INVARIANTS In Atiyah-Singer INDEX THEORY}

The Atiyah-Singer Index Theorem, first proved around 1963, equates the index, Ind $D=\operatorname{dim} \operatorname{Ker} D-\operatorname{dim}$ Coker $D$, of an elliptic operator $D: \Gamma(E) \rightarrow \Gamma(F)$ taking sections of a bundle $E$ over a closed manifold $M$ to sections of a bundle $F$ with $\left\langle P\left(M, \sigma_{\text {top }}(D)\right),[M]\right\rangle$, a characteristic number built from the topology of $M$ and topological information contained in the top order symbol of $D$ [5], [38]. For particular choices of $D$, this deep result encompasses Chern's generalization of the Gauss-Bonnet theorem, the Hirzebruch signature theorem, and Hirzebruch's generalization of the Riemann-Roch theorem, as well as giving many new results. Atiyah and Singer also proved the Families Index Theorem [6] for a family of elliptic operators $\left\{D_{n}\right\}$ parametrized by $n$ in a compact manifold $N$. This theorem identifies the Chern character of the index bundle IND $D$ in $H^{*}(N ; \mathbf{Q})$ with a characteristic class on $N$ built from the topology of $N$ and the pushforward of the symbols of the $D_{n}$; the significance of this theorem is that the Chern character of a bundle determines the K-theory isomorphism class of the bundle up to torsion. Here IND $D=\operatorname{KeR} D$ - COKER $D$ is a virtual bundle whose fiber for generic $n \in N$ is the formal difference KER $D_{n}-$ COKER $D_{n}$. For example, for a family of Dirac operators associated to a family of metrics $g_{n}$ on $M$ parametrized by $n \in N$, we get $\operatorname{ch}(\operatorname{IND} D)=\pi_{*} \hat{A}(M / N)$, where $\hat{A}(M / N)$ is the $\hat{A}$-polynomial of the bundle over $N$ whose fiber at $n$ is the bundle of spinors associated to $g_{n}$, and $\pi: M \times N \rightarrow N$ is

1991 Mathematics Subject Classification. Primary 58G25; Secondary 58G10, 58G25, 58G26. Partially supported by the NSF. 
the projection. This example becomes less trivial if we replace $M \times N$ by a manifold $X$ fibered by $M$ (cf. $\S 4)$.

Among the basic examples of elliptic operators are the so-called geometric operators, i.e. operators canonically associated to a Riemannian metric on $M$. For these operators, Hodge theory identifies the index with the Euler characteristic of de Rham-type cohomology groups. For example, the Gauss-Bonnet operator is given by $D=d+d^{*}$ taking even forms to odd forms, where $d$ is exterior differentiation and $d^{*}$ is its adjoint with respect to the Hodge inner product on forms induced by the metric. The index of $D$ is the Euler characteristic $\chi(M)$. The Dolbeault operator $\bar{\partial}+\bar{\partial}^{*}$, the analog of the Gauss-Bonnet operator for a complex Kähler manifold, has the arithmetic genus as its index, and the signature operator has $\sigma(M)$, the signature of $M$, as its index. (The index of the Dirac operator on a spin manifold does not have a Hodge theoretic topological interpretation.)

The simplest geometric example is the Gauss-Bonnet operator on a closed surface. The characteristic number given by the Index Theorem is $\langle e(M),[M]\rangle$, the value of the Euler class of $T M$ evaluated on the fundamental class of $M$. By Chern-Weil theory, this characteristic number equals $(1 / 2 \pi) \int_{M} K d A$, the integral of the Gaussian curvature. Thus the Index Theorem reduces in this case to the classical Gauss-Bonnet theorem: $\chi(M)=(1 / 2 \pi) \int_{M} K d A$. In particular, we have expressed the index as the integral of a local invariant, a top dimensional form (or density) computed pointwise canonically from the metric (i.e. independent of choice of chart).

This derivation of the Gauss-Bonnet theorem is somewhat indirect, as it uses Hodge theory to equate the analytic term, the index, with a topological expression, and then appeals to Chern-Weil theory to obtain a geometric expression for the topological side of the Index Theorem. Similar remarks apply to other geometric operators. Beginning with [1], heat equation proofs of the Index Theorem were developed which expressed the index of a geometrically defined operator directly as the integral of the correct Chern-Weil expression. In brief, it is easily shown that the supertrace $\operatorname{tr}_{s} e^{-t \mathbf{D}^{*} \mathbf{D}}=\operatorname{tr}\left(e^{-t D^{*} D}\right)-\operatorname{tr}\left(e^{-t D D^{*}}\right)$ of the heat operator is time independent, with $\mathbf{D}=D+D^{*}$ acting on sections of $E \oplus F$. As $t \rightarrow \infty$, the supertrace converges to the index of $D$ by a Hodge theory argument. By parabolic regularity theory, the heat operator $e^{-t \mathbf{D}^{*} \mathbf{D}}$ has a smooth integral kernel. The pointwise supertrace of the integral kernel of $e^{-t \mathbf{D}^{*} \mathbf{D}}$ is nonlocal, as it is built from the eigensections of $\mathbf{D}^{*} \mathbf{D}$, which are solutions of a global equation. However, by more or less standard pseudodifferential operator techniques, the pointwise supertrace has the asymptotic expansion

$$
\operatorname{tr}_{s} e^{-t \mathbf{D}^{*} \mathbf{D}}(t, x, x)=P(x)+\mathrm{O}(t),
$$

as $t \rightarrow 0$, for some local curvature invariant $P(x)=P(x, g)$, where $g$ is the Riemannian metric. Equating the long and short time behavior gives Ind $D=\int_{M} P(x)$ dvol.

From the complicated construction of the asymptotics, the curvature invariant $P(x)$ appears to contain many covariant derivatives of the curvature, while Chern-Weil forms contain no such derivatives. Either the nontrivial invariant theory developed by Atiyah-Bott-Patodi [1] and Gilkey [25], or the later, more direct "supersymmetric" approach of Getzler [24] and Patodi [40] shows that the covariant derivative terms vanish and identifies $P(x)$ dvol with the expected Chern-Weil form: the Euler form for the Gauss-Bonnet operator, the Todd class for the Dolbeault operator, Hirzebruch's L-polynomial for the signature operator, and the $\hat{A}$-polynomial 
for the Dirac operator. These results are sometimes called local index theorems; proofs of various cases are in [8], [44], [47]. As explained below, the geometric operators all have twisted versions formed by coupling the operator to a connection on an auxiliary bundle. The heat equation proof extends to the twisted case, and by K-theory arguments the full Index Theorem reduces to the cases of either twisted signature operators or twisted Dirac operators.

Bismut [9] and others extended Getzler's techniques to give similar local proofs of the Families Index Theorem. Here the Chern character as a differential form constructed by Chern-Weil theory from a connection on the index bundle is identified with the differential form version of the right hand side of the Families Index Theorem; in particular, the topological pushforward is refined to its smooth ana$\log$, integration along the fiber. These proofs are based on the superconnection techniques introduced in [42].

Although not as popular as the heat equation approach, one can prove the Index Theorem using the zeta functions $\zeta^{ \pm}(s)$ of $\Delta^{+}=D^{*} D, \Delta^{-}=D D^{*}$. Here $\zeta^{ \pm}(s)=$ $\sum\left(\lambda_{i}^{ \pm}\right)^{-s}$, where $\left\{\lambda_{i}^{ \pm}\right\}$are the nonzero eigenvalues of $\Delta^{ \pm}$counted with multiplicity. The smoothness of the heat kernel gives the convergence of its trace $\sum e^{-\lambda_{i}^{ \pm} t}$, which implies a Weyl-type estimate on the growth of the $\lambda_{i}^{ \pm}$. This in turn implies the convergence of $\zeta^{ \pm}(s)$ for $\operatorname{Re}(s)>>0$. Via a Mellin transform, the asymptotic expansion of the heat kernel gives a meromorphic continuation of $\zeta^{ \pm}(s)$ to all of $\mathbf{C}$ with at most simple poles at prescribed integers (or half-integers) [48]. In short, one obtains $\zeta^{+}(0)=\operatorname{dim} \operatorname{Ker} D-\int_{M} P^{+}(x)$ dvol for some complicated local invariant $P^{+}(x)$, and similarly for $\zeta^{-}(0)$. It is easy to show that $\zeta^{+}(0)=\zeta^{-}(0)$, and then supersymmetry arguments as above yield $P^{+}(x)-P^{-}(x)=P(x)$, from which the Index Theorem follows.

In analogy with number theoretic zeta functions, the special values or residues of the poles of the zeta function have geometric significance-e.g. $\zeta^{ \pm}(\operatorname{dim} M / 2)$ is a multiple of the volume of $M$. Moreover, the zeta functions associated to locally symmetric spaces often coincide with number theoretic zeta functions. However, this is as far as the analogy goes. For example, it is easy to check that the zeta function for the Laplacian on the simplest closed manifold, the circle, is a slight variation of the Riemann zeta function, so it is not a good idea to ask a geometer if the Riemann hypothesis holds for $\zeta^{ \pm}(s)$.

In general, zeta functions associated to a Riemannian metric do not have a functional equation, the main tool for studying arithmetic zeta functions. On the other hand, geometers can study the metric dependence of the value of a zeta function, a technique usually unavailable to number theorists due to rigidity results. This variational approach to special values of zeta functions and the analogous Lseries is a central theme in the subject.

\section{NonlocAl INVARIANTS - THE ETA INVARIANT}

The Gauss-Bonnet theorem for a surface with boundary picks up a correction term, the integral of the geodesic curvature over the boundary. Chern's generalization of Gauss-Bonnet to higher dimensions similarly has a term given by integrating a local invariant over the boundary. (The boundary term involves the geometric placement of the boundary in the manifold, and so cannot be expressed simply by a Chern-Weil-type curvature expression.) 
However, other examples of the Index Theorem do not admit such simple generalizations. In particular, assume the signature operator admitted a local correction term as above: $\sigma(M)=\int_{M} P_{\text {sig }}(x, g)+\int_{\partial M} Q \operatorname{sig}(x, g)$. If $\pi: M^{\prime} \rightarrow M$ is a $k$ to 1 finite covering, then with respect to the pullback metric $g^{\prime}$ of $g$, we would have $P_{\operatorname{sig}}\left(y, g^{\prime}\right)=P_{\operatorname{sig}}(\pi y, g)$, and similarly for $Q$. This implies $\sigma\left(M^{\prime}\right)=k \cdot \sigma(M)$. However, this formula is false in general.

Nevertheless, the dependence of the error term $\eta=\sigma(M)-\int_{M} P_{\text {sig }}(x, g)$ on the metric $g$ is a local invariant. For if $g_{t}$ is a curve of metrics, then by Chern-Weil theory $\left.(d / d t)\right|_{t=0} \eta^{g_{t}}$ is a local invariant. Of course, it is highly nontrivial to identify $\eta$ from this information.

In [3], Atiyah, Patodi and Singer gave the correction term for the Index Theorem for manifolds with boundary. If we assume for simplicity that the metric splits as a product on a collar of the boundary, the signature operator splits into $D=d / d t+A$, where $t$ is the collar parameter, $A= \pm(* d-d *)$ acting on all forms on the boundary, and $*$ is the Hodge star. The correction term depends only on $\partial M$, so with hindsight it is reasonable to guess that this term is built from the spectrum of the first order, selfadjoint elliptic operator $A$. Since $A$ has an infinite number of positive and negative eigenvalues, neither the heat operator nor the zeta function of $A$ is defined. However, in analogy with number theoretic L-series, we can define the eta function of $A$ by

$$
\eta(s)=\sum_{i} \frac{\operatorname{sgn}\left(\lambda_{i}\right)}{\left|\lambda_{i}\right|^{s}},
$$

where the sum is over the nonzero eigenvalues of $A$. By a simple scaling argument, the correction term must be $\eta(0)$ if the correction is a spectral invariant of $A$.

By a Mellin-type transform, it is possible to show that $\eta(s)$ converges for $\operatorname{Re}(s)$ $>>0$ and has a meromorphic continuation to $\mathbf{C}$ with $\eta(0)$ finite. However, note that the eta invariant does not vary smoothly with the metric, as a nonzero eigenvalue of $A=A_{t}$ may pass through zero as $t$ varies, causing a discontinuity in $\eta(s)$. So the correction term in general must keep track of the dimension of Ker $A$.

We now state the Index Theorem for manifolds with boundary in the case of the signature operator:

Theorem 2.1 (APS Theorem). Let $M$ be a manifold with boundary, and let $L(x, g)$ be the Chern-Weil representative of the Hirzebruch L-polynomial associated to a Riemannian metric $g$ on $M$. Assume the metric splits as a product near the boundary. Then

$$
\sigma(M)=\int_{M} L(x, g)-\eta(0) .
$$

This theorem shows that the real valued eta invariant $\eta(0)$ is nonlocal. There are similar results for other geometric operators. If the metric is not a product near the boundary, the right hand side of the last equation also contains the integral of a local invariant over $\partial M$, as in the Chern-Gauss-Bonnet theorem. The text [33], which contains extensive references, gives a proof of the APS theorem using Getzler's methods, in which the eta invariant appears naturally in the course of the proof. Finally, we note that the nonlocal nature of the eta invariant is reflected in the fact that the APS theorem can be proved by considering a boundary value problem for $\mathbf{D}^{*} \mathbf{D}$ with respect to nonlocal boundary conditions. The index of $D$ is not quite equal to $\sigma(M)$, but it involves a term which cancels with the dimension of $\operatorname{Ker} A$, so this discontinuous term does not appear in the APS theorem. 
Needless to say, computing the eta invariant is quite difficult. However, the fact that the variation of the eta invariant is local leads to a computable invariant. For let $E$ be a hermitian bundle over $M$ with a flat unitary connection $\nabla$. Form the coupled signature operator $D_{\nabla}$ acting on $E$-valued forms by $D_{\nabla}=D_{\text {sig }} \otimes$ Id + Id $\otimes \nabla$. Since the bundle is flat, $D_{\nabla}$ locally looks like $k$ copies of $D_{\text {sig }}$, where $k=\operatorname{rank}(E)$. Let $g_{t}$ be a curve of metrics with variation $s=\left.(d / d t)\right|_{t=0} g_{t}$, a symmetric two-tensor. Since the variations of the eta invariants of $D_{\text {sig }}, D_{\nabla}$ are computed from the asymptotics of the associated heat operators, it is easy to see that the variations $\delta_{s}$ of the eta invariants satisfy $k \cdot \delta_{s} \eta_{D_{\text {sig }}}(0)=\delta_{s} \eta_{D_{\nabla}}(0)$. In particular, the difference $k \cdot \delta_{s} \eta_{D_{\text {sig }}}(0)-\delta_{s} \eta_{D_{\nabla}}(0)$ is independent of the Riemannian metric and hence is a smooth invariant of the manifold $M$ and the flat bundle $E$ (or equivalently, of the manifold $M$ and a unitary representation of $\pi_{1}(M)$ ). The Index Theorem for Flat Bundles [4], [26] gives a topological interpretation of this invariant.

\section{NONLOCAL INVARIANTS - THE DETERMINANT}

For geometric operators such as the Gauss-Bonnet and Dolbeault operator, the index is the Euler characteristic of certain cohomology groups. If these groups vanish, the Index Theorem has nothing to say, and secondary geometric and topological invariants appear. This is the case for Reidemeister torsion, which is (most easily) defined only when the cohomology of $M$ twisted by a unitary representation $\rho$ of $\pi_{1}(M)$ vanishes [34]. In particular, Reidemeister torsion is a "precohomological" invariant, in that it is defined from a twisted cochain complex; this torsion was originally introduced to distinguish lens spaces with isomorphic cohomology rings and homotopy groups.

Ray and Singer [43] noted that Reidemeister torsion is computed from a triangulation of $M$ as a linear combination of determinants of combinatorial Laplacians, and conjectured that the Reidemeister torsion equals the analytic torsion,

$$
T(M, g, \rho)=\frac{1}{2} \sum_{k=1}^{n}(-1)^{k} k \ln \operatorname{det} \Delta_{\rho}^{k},
$$

the corresponding combinations of determinants of Laplacians on $k$-forms with values in the flat bundle determined by $\rho$.

Of course, the product of the eigenvalues of these Laplacians is infinite, so the notion of determinant must be redefined, or regularized. Since the determinant of an invertible finite dimensional positive selfadjoint transformation $T$ can be computed from its eigenvalues $\left\{\lambda_{i}\right\}$ by the formula $-\ln \operatorname{det} T=\left.(d / d s)\right|_{s=0} \sum \lambda_{i}^{-s}$, Ray and Singer defined the determinant of a Laplacian-type operator to be $\exp \left(-\zeta^{\prime}(0)\right)$. In a similar way, $\zeta(0)$ regularizes the dimension of the domain of the Laplacian, and more importantly, the eta invariant regularizes the number of positive minus the number of negative eigenvalues of the operator $A$.

Based on the evidence presented in [43], Cheeger [21] and Müller [35] proved the equality of analytic and Reidemeister torsion. Cheeger's proof uses surgery techniques to reduce the problem to lens spaces, where the result was known, while Müller's proof examines the convergence of the spectral theory of the combinatorial Laplacians to that of the smooth Laplacians as the mesh of the triangulation goes to zero. Vishik [50] has given a cutting and pasting proof based on ideas from 
topological quantum field theory, and Bismut and Zhang [17] have a proof based on Witten's proof [52] of the Morse inequalities.

For a fixed Laplacian, such as the Laplacian on bundle-valued $k$-forms, $\zeta^{\prime}(0)$ is a subtle spectral invariant. In contrast to $\zeta(0)$, it is not a local invariant, as covering space examples show, and in contrast to $\eta(0)$, its variation is not a local invariant. This can be seen by computing examples on flat tori, where the zeta function is a Selberg-Chowla zeta function. Thus there is no index theorem for flat bundles for $\zeta^{\prime}(0)$. However, for operators with reasonable change under conformal change of the metric, $\zeta^{\prime}(0)$ is an invariant of the conformal class of a metric; examples include Dirac operators and conformal Laplacians [18], [39], [46] (cf. [37] for a proof of the uniformization theorem for Riemann surfaces based on the variation of $\zeta^{\prime}(0)$ ).

In direct contrast to the original Index Theorem, which only gives Ind $D=0$ if $\operatorname{dim} M$ is odd, Reidemeister/analytic torsion is nontrivial only if $\operatorname{dim} M$ is odd. Note the continued analogy with number theoretic zeta functions, where the residue or leading order term of a special value has significance, in that analytic torsion is independent of the metric only in the case where $\zeta(0)=0$.

\section{Relations AMONG THE InVARIANts}

All known relations among the local and nonlocal invariants involve the Families Index Theorem. The first relation uncovered involves the determinant line bundle of a family of operators, which is the easiest index bundle to understand topologically, but whose geometry is nontrivial.

Motivated by questions in quantum field theory, Atiyah and Singer [7], [49] studied the problem of canonically defining the determinants of a family of invertible first order elliptic operator $D: \Gamma(E) \rightarrow \Gamma(F)$ for different bundles $E, F$. If $D=D_{n}$ were a family of transformations, parametrized by $n \in N$, between finite dimensional vector spaces $V, W$, then defining the determinant of the family would be equivalent to picking a section of the line bundle

$$
\Lambda^{\max } V \otimes\left(\Lambda^{\max } W\right)^{*} \simeq \Lambda^{\max } \operatorname{Ker} D \otimes\left(\Lambda^{\max } \operatorname{Coker} D\right)^{*}
$$

over $N$. For the infinite dimensional vector spaces $\Gamma(E), \Gamma(F)$, only the right hand side of the last equation is defined, so defining the determinant of the family reduces to trivializing the determinant line bundle DET $D=\Lambda^{\max } \mathrm{KeR} D \otimes$ $\left(\Lambda^{\max } \text { CoKer } D\right)^{*}$ of the index bundle. Thus there are topological obstructions to the solution given by the Families Index Theorem. However, even if the bundle is trivial, there is no canonical trivialization, unless the bundle comes with a canonical nonvanishing section, or equivalently with a canonical flat connection with vanishing holonomy.

In [41], Quillen introduced a canonical hermitian metric on the determinant line bundle for the family of twisted Dolbeault operators over a Riemann surface. In brief, DET $D$ has a canonical section det $D$ which is locally given by $\operatorname{det} D=$ $v^{1} \wedge \ldots \wedge v^{k} \otimes\left(D v^{1} \wedge \otimes \ldots \wedge D v^{k}\right)^{*}$, where $\left\{v^{i}\right\}$ is an orthonormal basis of eigensections of $D^{*} D$ with eigenvalues $\lambda_{i}$ lying below some value $a$. The Quillen metric on DET $D$ is defined by

$$
\|\operatorname{det} D\|^{2}=\left(\prod \lambda_{i}\right) e^{-\zeta_{D^{*}, a}^{\prime}(0)},
$$

where the zeta function $\zeta_{D^{*} D, a}(s)$ is built from the eigenvalues above $a$. This formula is independent of the choice of $a$ and is motivated by the easy equality $\|\operatorname{det} D\|^{2}=\operatorname{det} D^{*} D$ for $D$ a transformation of finite dimensional vector spaces 
with $\left\|D^{*} D\right\|<a$. In Quillen's holomorphic example, DET $D$ has a canonical connection whose curvature is nonzero in general; thus there is a geometric obstruction to defining the determinant of the family of operators, even if the topological obstruction (the first Chern class of Det $D$ ) vanishes.

The holomorphic category is too rigid to handle the general case of twisted Dirac operators. In [13], Bismut and Freed defined an analogous connection on the determinant line bundle for a family of twisted Dirac operators and proved that the curvature of this connection, which (i) is a combination of second variations of $\zeta^{\prime}(0)$, and (ii) represents the first Chern class, is precisely the degree two component of the differential form in the local Families Index Theorem. In light of the nonlocal nature of the first variation of $\zeta^{\prime}(0)$, this localization of the curvature is especially striking.

Computing the holonomy of the Quillen metric is more difficult, as this involves integrating the connection one-form around loops in the base manifold. This is a nonlocal computation on a general bundle and is all the more nonlocal here, since the connection one-form is essentially the first variation of $\zeta^{\prime}(0)$. Of course, if the family of operators is constant, the holonomy is trivial. In [51], Witten gave a heuristic argument for the limit of the holonomy as the metric on the base manifold is scaled up; this so-called adiabatic limit corresponds to taking a limit as the operators get closer and closer to being constant.

The geometric framework for Witten's argument consists of a Riemannian submersion $\pi: X \rightarrow N$ of Riemannian manifolds with fiber diffeomorphic to the even dimensional closed manifold $M$. Assuming $X, M, N$ are spin, we have a family of Dirac operators $D_{n}, n \in N$, acting on positive spinors on $\pi^{-1}(n)$. (For simplicity, we will not discuss the twisted case.) The holonomy around a loop $\gamma \subset N$ is formally controlled by the phase of the determinant of the associated Dirac operator $\mathbf{D}$ on all spinors on $\pi^{-1}(\gamma)$. This phase is formally $(-1)$ to the number of negative eigenvalues of $\mathbf{D}$, but this number must of course be regularized. If $\mathbf{D}$ were a finite dimensional transformation, the number of negative eigenvalues would be exactly $\left(\zeta_{\mathbf{D}}(0)-\eta_{\mathbf{D}}(0)\right) / 2$, so in general $\exp (i \pi \eta(0))$ enters in the phase of the determinant. This informal argument was presented rigorously in [14], [22].

Theorem 4.1. Let $\{D\}=\left\{D_{n}\right\}$ be a family of Dirac operators, parametrized by $n$ in a compact Riemannian manifold $N$ as above. The curvature of the determinant line bundle DET $D$ is given by the degree two component of $\int_{M} \hat{A}\left(D_{n}, g\right)$, where $\hat{A}\left(D_{n}, g\right)$ is the $\hat{A}$-polynomial as a differential form associated to the family $\{D\}$ and a metric $g$ on $N$, and $\int_{M}$ denotes integration over the fiber. The holonomy of a loop $\gamma \subset N$ is $(-1)^{\text {Ind } \mathbf{D}} \exp (i \pi \boldsymbol{\eta})$, where $\boldsymbol{\eta}$ is the adiabatic limit of $\left(\eta_{\mathbf{D}}(0)+\right.$ $\operatorname{dim} \operatorname{Ker} \mathbf{D}) / 2$.

In summary, in the determinant line bundle picture, the integral of the first variation of $\zeta^{\prime}(0)$ around a loop relates the holonomy of the Quillen-Bismut-Freed connection to the eta invariant of a Dirac operator over the loop, while the Families Index Theorem computes the curvature, which depends on the second variation of $\zeta^{\prime}(0)$.

Certainly there is a loss of information in passing from the index bundle to the determinant line bundle. The relation between the eta invariant and the Families Index Theorem at the level of the index bundle has been made more precise in work of Bismut and Cheeger [10], [11]. For this theory let $\pi: X \rightarrow N$ be a Riemannian submersion with fiber diffeomorphic to the even dimensional manifold with boundary $M$. We assume that $X, N, M$ are spin and that the kernel of the 
Dirac operator $D=D_{n}$ restricted to the boundary of each fiber has constant rank. Then the local Families Index Theorem for Manifolds with Boundary is the equality of differential forms

$$
\operatorname{ch}(\operatorname{IND} D)=\int_{M} \hat{A}\left(D_{n}, g\right)-\tilde{\eta},
$$

where the eta form $\tilde{\eta}$ is an even dimensional form on $N$ canonically constructed from $\left.D\right|_{\partial X}$, and $g$ is the metric on $X$. (There is a similar result if $M$ is odd dimensional.) In particular, the degree zero component of $\tilde{\eta}$ satisfies $\tilde{\eta}_{0}(n)=\eta(0)$, the eta invariant of $D_{n}$, and as such this Index Theorem is a strong generalization of the APS theorem.

In addition, $\tilde{\eta}$ satisfies the transgression formula on $\partial X$

$$
d \tilde{\eta}=\operatorname{ch}\left(\text { IND } D_{\partial M}\right)-\int_{\partial M} \hat{A}\left(D_{\partial X},\left.g\right|_{\partial X}\right)
$$

when $M$ is odd dimensional and the dimension of $\operatorname{Ker} D_{\partial M}$ is constant. Since the right hand side is a local invariant, the transgression formula generalizes the result that the variation of the eta invariant is local. The corresponding formula for $M$ even dimensional simply reads $d \tilde{\eta}=0$, so $\tilde{\eta}$ determines an even dimensional cohomology class in $N$ associated directly to $\partial X$.

In later work [16], Bismut and Lott have proved an analogous theorem which makes precise the sense in which analytic torsion appears as a secondary invariant once both sides of the index theorem vanish a priori. Let $E$ be a flat bundle over $X$, where as above we have the Riemannian submersion $\pi: X \rightarrow N$ with fiber $M$. For $k$ odd, let $c_{k}(E) \in H^{k}(X ; \mathbf{R})$ denote the Kamber-Tondeur classes of $E$ [29]; these classes have a Chern-Weil description given below. Let $e(T M / N)$ be the Euler class of $T M / N$, the tangent bundle along the fibers. Let $H^{p}\left(M ;\left.E\right|_{M}\right)$ denote the flat vector bundle over $N$ whose fiber at $n \in N$ is the cohomology group $H^{p}\left(M_{n} ;\left.E\right|_{M_{n}}\right)$, where $M_{n}=\pi^{-1}(n)$. Then we have the following cohomological result.

Theorem 4.2. For any odd integer $k$,

$$
\pi_{*}\left(e(T M / N) c_{k}(E)\right)=\sum_{p=0}^{\operatorname{dim} M}(-1)^{p} c_{k}\left(H^{p}\left(M ;\left.E\right|_{M}\right)\right)
$$

as classes in $H^{k}(N ; \mathbf{R})$.

This theorem is a $C^{\infty}$ (global) analogue of a (local) version of the GrothendieckRiemann-Roch theorem for holomorphic submersions due to Bismut, Gillet and Soulé [15], and as such is a families-type index theorem.

The striking feature of the proof of Theorem 3 is its local, geometric nature; a global, topological version appeared subsequently [23]. To state the more precise geometric version, pick a hermitian metric $h$ on $E$. Since $E$ is flat with respect to a connection $\nabla^{E}$, locally there exists a basis $\left\{e_{i}\right\}$ of the fibers of $E$ with $\nabla^{E} e_{i}=0$. With respect to this basis, $h$ is a function on $X$ with values in Hermitian matrices. Define an $\operatorname{End}(E)$-valued one-form on $X$ by $\omega(E, h)=h^{-1} d h$. For odd positive integers $k$, set

$$
c_{k}(E, h)=(2 \pi i)^{-(k-1) / 2} 2^{-k} \operatorname{Tr}\left(\omega^{k}(E, h)\right),
$$

where $\omega^{k}$ is $\omega$ wedged with itself $k$ times. The $k$-form $c_{k}(E, h)$ is closed and its cohomology class equals $c_{k}(E)$. Let $\Omega$ be the curvature of a unitary connection $\nabla$ on $T M / N$, and let $e(\Omega)$ be the Chern-Weil representative of $e(T M / N)$. The 
fibers $H^{p}\left(M_{n} ;\left.E\right|_{M_{n}}\right)$ inherit an inner product $h^{H^{p}}$ from the $L^{2}$ inner product on $\Gamma\left(T M /\left.N_{n} \otimes E\right|_{M_{n}}\right)$, and we denote the associated Chern-Weil representative of $c_{k}\left(H^{p}\left(M ;\left.E\right|_{M}\right)\right)$ by $c_{k}\left(H^{p}\left(M ;\left.E\right|_{M}\right), h^{H^{p}}\right)$. Recall that $g$ is the metric on $X$.

Theorem 4. There exist $(k-1)$-forms

$$
\mathcal{T}_{k-1}=\mathcal{T}_{k-1}(g, h)
$$

on $N$ such that

(i) for any odd integer $k$,

$$
d \mathcal{T}_{k-1}=\int_{M} e(\Omega) c_{k}(E, h)-\sum_{p=0}^{\operatorname{dim} M}(-1)^{p} c_{k}\left(H^{p}\left(M ;\left.E\right|_{M}\right), h^{H^{p}}\right)
$$

as differential $k$-forms;

(ii) if the cohomology groups $H^{p}\left(M_{n} ;\left.E\right|_{M_{n}}\right)$ vanish for all $p$ and all $n \in N$, and if $M$ is odd dimensional, then the forms $\mathcal{T}_{k-1}$ are closed, and the class $\left[\mathcal{T}_{k-1}\right] \in$ $H^{k-1}(N ; \mathbf{R})$ is independent of the choices of $g, h$. In particular, $\left[\mathcal{T}_{0}\right]$ is (represented by) the locally constant function which is half the analytic/Reidemeister torsion of the pair $\left(M_{n},\left.E\right|_{M_{n}}\right)$.

This result has several striking features. First, it has Theorem 3 as an immediate corollary. Second, it realizes the analytic/Reidemeister torsion as the degree zero component of a sophisticated local families-type index theorem. Third, note that the first statement in (ii) follows immediately from (i), since $\operatorname{dim} M$ odd implies $\int_{M} e(\Omega) c_{k}(E, h)$ is an even form and so has no component in degree $k$. Thus, as expected, the so-called higher torsion forms $\mathcal{T}_{k-1}$ have cohomological significance only when the cohomological information in the index-type Theorem 3 is trivial.

Of course, one expects that the cohomology classes of the higher torsion forms should equal some combinatorially defined "higher Reidemeister torsion". Such a torsion has been defined by Igusa and Klein [28], [30], and the two higher torsions agree in the few cases where they have been computed. At present, however, the equality in full generality is unknown.

Recently, Heitsch and Lazarov [27] have produced higher torsion forms for bundles over foliated compact manifolds. This construction encompasses Theorem 4 as well as classical analytic torsion and its generalizaton to noncompact covering spaces [32]. At present, no analogous topological construction has been given.

In a different direction, Lott [31] has used an extension $\widetilde{\mathcal{T}}$ of the higher torsion forms to study $\pi_{i}(\operatorname{Diff}(Z)) \otimes \mathbf{Q}$, where $\operatorname{Diff}(Z)$ denotes the diffeomorphism group of a closed smooth $K(\Gamma, 1)$ manifold $Z$. For a given $\alpha: S^{i} \rightarrow \operatorname{Diff}(Z)$, the standard clutching construction of gluing two $(i+1)$-balls times $Z$ along their boundaries via $\alpha$ produces a manifold $M_{\alpha}$ fibering over $S^{i+1}$ with fiber $Z$. Under certain technical hypotheses, $\int_{S^{i+1}} \widetilde{\mathcal{T}}\left(M_{\alpha}\right)$ lies in $H_{*}(\Gamma ; \mathbf{C})$. In fact, there is an explicit conjectured formula for $\pi_{i}(\operatorname{Diff}(Z)) \otimes \mathbf{Q}$ in terms of $H_{k}(\Gamma, \mathbf{C})$. Lott's map $\int_{S^{i+1}} \widetilde{\mathcal{T}}(M):. \pi_{i}(\operatorname{Diff}(Z)) \rightarrow H_{*}(\Gamma ; \mathbf{C})$ gives the first analytic evidence for this old conjecture (which is known in several cases), although at this point it is not known if this map is the desired isomorphism. In contrast, the homeomorphism group of $Z$ has vanishing rational homotopy groups, so it seems natural to approach the conjecture via a smooth, i.e. analytic, construction.

In summary, strong relations between the nonlocal invariants and classical index theory are now firmly established. These relations have applications to algebraic K-theory indicated in [16], [23], [31], as well as to the subject of index theory on 
open manifolds (e.g. [2], [12], [19], [20], [32], [36], [45]), that go beyond the limits of this article.

\section{REFERENCES}

1. M. F. Atiyah, R. Bott, and V. K. Patodi, On the heat equation and the index theorem, Inventiones Math. 19 (1973), 279-330. MR 58:31287

2. M. F. Atiyah, H. Donnelly, and I. M. Singer, Eta invariants, signature defects of cusps and values of L-functions, Ann. Math. 118 (1983), 131-177. MR 86g:58134a

3. M. F. Atiyah, V. K. Patodi, and I. M. Singer, Spectral asymmetry and Riemannian geometry. I, Math. Proc. Camb. Phil. Soc. 77 (1975), 43-69. MR 53:1655a

4. , Spectral asymmetry and Riemannian geometry. III, Math. Proc. Camb. Phil. Soc. 79 (1976), 71-99. MR 53:1655c

5. M. F. Atiyah and I. M. Singer, The index of elliptic operators on compact manifolds, Bull. Amer. Math. Soc. 69 (1963), 422-433. MR 28:626

6. $ـ$ The index of elliptic operators. III, Ann. Math. 87 (1968), 546-604. MR 38:5245

7. Dirac operators coupled to vector potentials, Proc. Nat. Acad. Sci., USA 81 (1984), 2597-2600. MR 86g:58127

8. N. Berline, E. Getzler, and M. Vergne, Heat Kernels and Dirac Operators, Grundlehren der mathematischen Wissenschaften, vol. 298, Springer-Verlag, Berlin, 1992. MR 94e:58130

9. J.-M. Bismut, The Atiyah-Singer index theorem for families of Dirac operators: two heat equation proofs, Inventiones Math. 83 (1986), 91-151. MR 87g:58117

10. J.-M. Bismut and J. Cheeger, Families index for manifolds with boundary, superconnections, and cones. I. Families of manifolds with boundary and Dirac operators, J. Funct. Anal. 89 (1990), 313-363. MR 91e:58180

11. Chern character, J. Funct. Anal. 90 (1990), 306-354. MR 91e:58181

12. _ Transgressed Euler classes of $S L(2 n, Z)$ vector bundles, adiabatic limits of eta invariants and special values of L-functions, Ann. scient. Éc. Norm. Sup. 25 (1992), 335-391. MR 94e:57042

13. J.-M. Bismut and D. Freed, The analysis of elliptic families I: Metrics and connections on determinant line bundles, Commun. Math. Phys. 106 (1986), 159-176. MR 88h:58110a

14. _ The analysis of elliptic families II: Dirac operators, eta invariants, and the holonomy theorem of Witten, Commun. Math. Phys. 107 (1986), 103-163. MR 88h:58110b

15. J.-M. Bismut, H. Gillet, and C. Soulé, Analytic torsion and holomorphic determinant bundles. I-III, Commun. Math. Phys. 115 (1988), 49-78, 79-126, 301-351. MR 89g:58192a/b/c

16. J.-M. Bismut and J. Lott, Flat vector bundles, direct images, and higher real analytic torsion, J. Amer. Math. Soc. 8 (1995), 291-363. MR 96g:58202

17. J.-M. Bismut and W. Zhang, An extension of a theorem of Cheeger and Müller, Astérisque 205 (1992), 3-235. MR 93j:58138

18. T. Branson and B. Ørsted, Conformal geometry and global invariants, Diff. Geom. Appl. 1 (1991), 279-308. MR 94k:58154

19. D. Burghelea, L. Friedlander, and T. Kappeler, Torsions for manifolds with boundary and gluing formulas, preprint.

20. A. Carey and V. Mathai, $L^{2}$-acyclicity and $L^{2}$-torsion invariants, Contemporary Mathematics 105 (1990), American Mathematical Society, Providence, RI, pps. 91-118. MR 91e:58187

21. J. Cheeger, Analytic torsion and the heat equation, Ann. Math. 109 (1979), 259-322. MR 80j:58065a

22. X. Dai and D. Freed, $\eta$-invariants and determinant lines, J. Math. Phys. 35 (1994), 51555194. MR 96a:58204

23. W. Dwyer, M. Weiss, and B. Williams, A parametrized index theorem for the algebraic $K$ theory Euler class, preprint, http:/www.math.uiuc.edu/K-theory/0086/(1995).

24. E. Getzler, A short proof of the local Atiyah-Singer Index Theorem, Topology 25 (1986), 111-117. MR 87h:58207

25. P. B. Gilkey, Curvature and the eigenvalues of the Laplacian for elliptic complexes, Adv. in Math. 10 (1973), 344-381. MR 48:3081

26. _ Invariance Theory, the Heat Equation, and the Atiyah-Singer Index Theorem, Publish or Perish, Wilmington, DE, 1984. MR 86j:58144 
27. J. Heitsch and C. Lazarov, Riemann-Roch-Grothendieck and torsion for foliations, preprint.

28. K. Igusa, Parametrized Morse theory and its applications, Proc. Int. Cong. Math., Kyoto 1990, Mathematical Society of Japan, Tokyo (1991), 643-651. MR 93c:57022

29. F. Kamber and P. Tondeur, Characteristic invariants of foliated bundles, Manuscripta Math. 11 (1974), 51-89. MR 48:12556

30. J. Klein, Higher Franz-Reidemeister torsion: low-dimensional applications, Contemporary Mathematics 150 (1993), American Mathematical Society, Providence, RI, pps. 195-204. MR 94g:19004

31. J. Lott, Diffeomorphisms, analytic torsion and noncommutative geometry, dg-ga/9607006.

32. , Heat kernels on covering spaces and topological invariants, J. Differential Geometry 35 (1992), 471-510. MR 93b:58140

33. R. B. Melrose, The Atiyah-Patodi-Singer Index Theorem, A. K. Peters, Wellesley, MA, 1993. MR 96g: 58180

34. J. Milnor, Whitehead torsion, Bull. Amer. Math. Soc. 72 (1966), 358-426. MR 33:4922

35. W. Müller, Analytic torsion and R-torsion of Riemannian manifolds, Adv. in Math. 28 (1978), 233-305. MR 80j:58065b

36. $-L^{2}$ index theory, eta invariants and values of $L$-functions, Contemporary Mathematics 105 (1990), American Mathematical Society, Providence, RI, pps. 141-190. MR 91g:58274

37. B. Osgood, R. Phillips, and P. Sarnak, Extremals of determinants of Laplacians, J. Funct. Anal. 80 (1988), 148-211. MR 90d:58159

38. R. Palais (ed.), Seminar on the Atiyah-Singer Index Theorem, Annals of Mathematics Study, vol. 57, Princeton University Press, Princeton, 1965. MR 33:6649

39. T. Parker and S. Rosenberg, Invariants of conformal Laplacians, J. Differential Geometry 25 (1987), 199-222. MR 89e:58118

40. V. K. Patodi, An analytic proof of the Riemann-Roch-Hirzebruch theorem for Kähler manifolds, J. Differential Geometry 5 (1971), 251-283. MR 44:7502

41. D. Quillen, Determinants of Cauchy-Riemann operators on Riemann surfaces, Funk. Anal. i Prilozhen 19 (1985), 37-41. MR 86g:32035

42. _ Superconnections and the Chern character, Topology 24 (1985), 89-95. MR 86m: 58010

43. D. B. Ray and I. M. Singer, R-torsion and the Laplacian on Riemannian manifolds, Adv. in Math. 7 (1971), 145-210. MR 45:4447

44. J. Roe, Elliptic Operators, Topology, and Asymptotic Methods, Pitman Research Notes in Mathematics, vol. 179, Longman Scientific and Technical, Burnt Mill, UK, 1988. MR 89j:58126

45. C Coarse cohomology and index theory on complete Riemannian manifolds, Memoirs of the Amer. Math. Soc., 497, 1993. MR 94a:58193

46. S. Rosenberg, The determinant of a conformally covariant operator, J. London Math. Soc. 36 (1987), 553-568. MR 89h:58205

47. — The Laplacian on a Riemannian Manifold, Cambridge U. Press, Cambridge, UK, 1997.

48. R. Seeley, Complex powers of an elliptic operator, Proc. Symp. Pure Math., vol. 10, Amer. Math. Soc., Providence, RI, 1967, pp. 288-307. MR 38:6220

49. I. M. Singer, The $\eta$-invariant and the index, Mathematical Aspects of String Theory (S. T. Yau, ed.), World Scientific Press, Singapore, 1987, pp. 239-258. CMP 20:04

50. S. Vishik, Generalized Ray-Singer conjecture. I. A manifold with a smooth boundary, Commun. Math. Phys. 167 (1995), 1-102. MR 96f:58184

51. E. Witten, Global gravitational anomalies, Commun. Math. Phys. 100 (1985), 197-229. MR $87 \mathrm{k}: 58282$

52. _ Supersymmetry and Morse theory, J. Differential Geometry 17 (1982), 661-692. MR 84b:58111

Department of Mathematics, Boston University, Boston, Massachusetts 02215

E-mail address: sr@math.bu.edu 